Provided for non-commercial research and education use. Not for reproduction, distribution or commercial use.

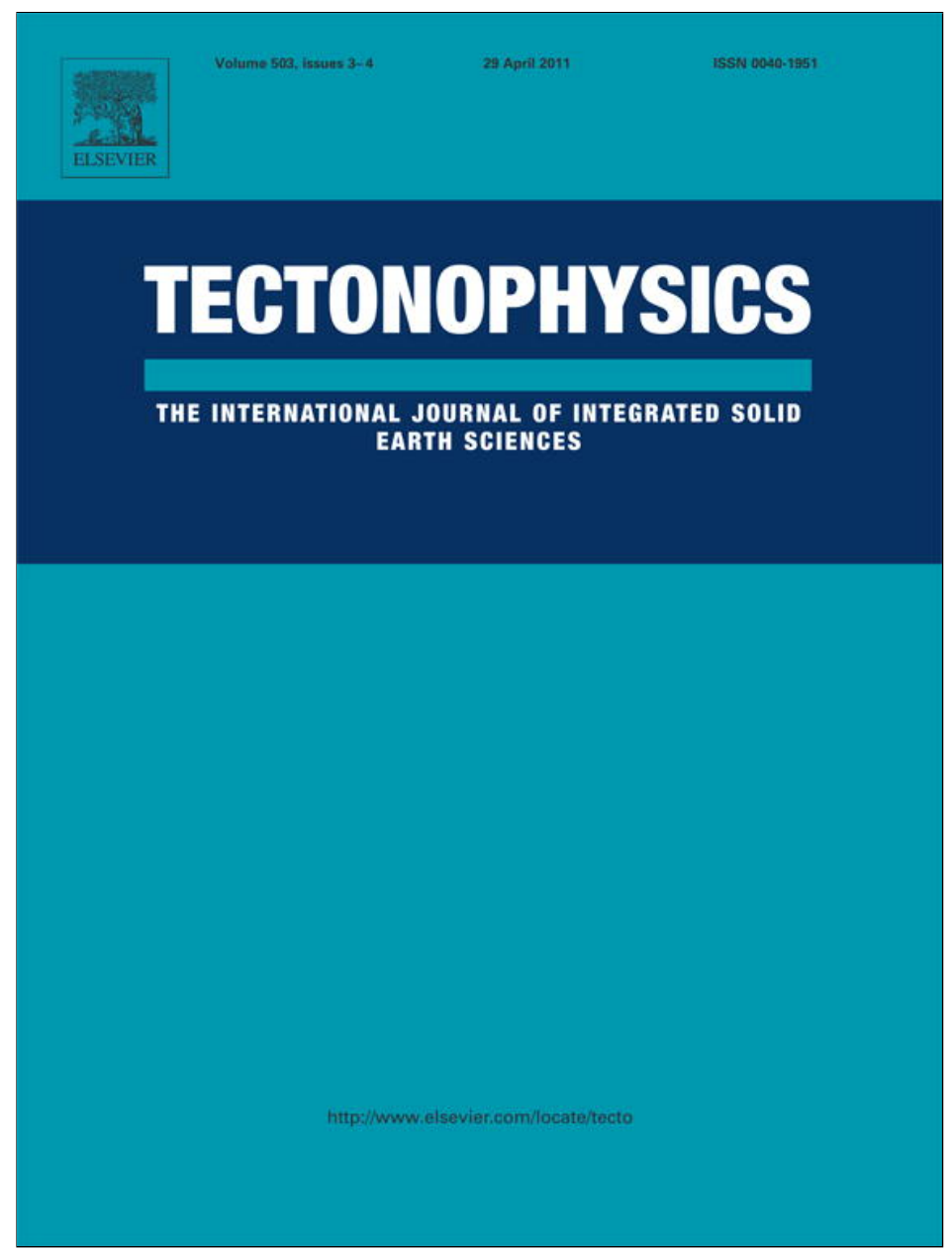

This article appeared in a journal published by Elsevier. The attached copy is furnished to the author for internal non-commercial research and education use, including for instruction at the authors institution and sharing with colleagues.

Other uses, including reproduction and distribution, or selling or licensing copies, or posting to personal, institutional or third party websites are prohibited.

In most cases authors are permitted to post their version of the article (e.g. in Word or Tex form) to their personal website or institutional repository. Authors requiring further information regarding Elsevier's archiving and manuscript policies are encouraged to visit:

http://www.elsevier.com/copyright 


\title{
Identifying long-range correlated signals upon significant periodic data loss
}

\author{
P.A. Varotsos *, N.V. Sarlis, E.S. Skordas \\ Solid State Section and Solid Earth Physics Institute, Physics Department, University of Athens, Panepistimiopolis, Zografos 157 84, Athens, Greece
}

\section{A R T I C L E I N F O}

\section{Article history:}

Received 27 May 2010

Received in revised form 17 January 2011

Accepted 27 February 2011

Available online 3 March 2011

Keywords:

Seismic electric signals

Detrended fluctuation analysis

Geoelectrical field measurements

Long-range correlated signals

\begin{abstract}
A B S T R A C T
When monitoring geophysical parameters, data from segments that are contaminated by noise may have to be abandoned. This is the case, for example, in the geoelectrical field measurements at some sites in Japan, where high noise - due mainly to leakage currents from DC driven trains - prevails almost during 70\% of the 24 hour operational time. We show that even in such a case, the identification of seismic electric signals (SES), which are long-range correlated signals, may be possible, if the remaining noise free data are analyzed in natural time along with detrended fluctuation analysis (DFA).
\end{abstract}

(c) 2011 Elsevier B.V. All rights reserved.

\section{Introduction}

In many cases of geophysical and/or geological interest, it happens that for substantial parts of the time of data collection, high noise prevents any attempt for extracting a useful signal. Data for such time segments are removed from further analysis. The appearance of such a noise may be periodic as in the case treated in the present work. It is the objective of this paper to examine whether the remaining data allow the identification of long-range temporal correlations.

The present study was motivated from the results of geoelectrical measurements in Japan aiming at the detection of Seismic Electric Signals (SES), which are low-frequency $(\leq 1 \mathrm{~Hz})$ variations of the electric field of the earth that precede earthquakes (Varotsos and Alexopoulos, 1984a,b). SES sometime appears as a single signal lasting for minutes but often many SES (hereafter called "pulses" as needed) keep appearing during certain length of time, which may be as long as a few days or more. Such a case is called SES activity. It has been shown that the SESs in a SES activity have long range temporal correlations characteristic to critical phenomena (Varotsos et al., 2002). The measurements in Japan have detected clear SES either at noise-free measuring sites or at noisy stations when the SES happened to occur at midnight, i.e., when the noise level was low (Uyeda et al., 2000, 2002). The major difficulty at many sites is the contamination of records by high noise due to leakage currents from DC driven trains and other artificial sources, against which some countermeasure such as independent component analysis to extract signals has been attempted (e.g., see Orihara et al. 2009). The low noise time occurs from 00:00 to

\footnotetext{
* Corresponding author. Tel.: +30 2109617573 ; fax: +30 2109601721. E-mail address: pvaro@otenet.gr (P.A. Varotsos).
}

06:00 and from 22:00 to 24:00 local time (LT) when nearby DC driven trains cease service, i.e., almost only $30 \%$ of the $24 \mathrm{~h}$. Thus, the question arises whether it is still possible to identify SES upon removing the noisy data segments lasting for the period 06:00 to 22:00 every day. The answer to this question is attempted in this paper for the case when the duration of SES activity is much longer compared to those of individual pulses, i.e., a few days to a few weeks or even more, although admittedly long lasting SES activity is rather seldom, limiting the applicability of the results described below.

The key point in the present work is the use of the following two modern methods: The natural time analysis of the remaining data and the detrended fluctuation analysis (DFA). The present question differs from the one in which we investigated (Skordas et al., 2010) the effect of the random in time removal of data segments of fixed length on the scaling properties of SES activities. It also differs from the case in which the lengths of the lost or removed data segments are random and may follow a certain type of distribution (Ma et al., 2010).

We now briefly describe the time series analysis in natural time $\chi$, which is a new time domain (Varotsos, 2005; Varotsos et al., 2002, 2003a,b;). In a time series comprising $\mathrm{N}$ events, the natural time $\chi_{\mathrm{k}}=\mathrm{k} / \mathrm{N}$ serves as an index for the occurrence of the k-th event. The evolution of the pair $\left(\chi_{k}\right.$ and $\left.Q_{k}\right)$ is studied, where $Q_{k}$ is a quantity proportional to the energy released in the k-th event. For dichotomous signals, which is frequently the case of $S E S$ activities, the quantity $Q_{k}$ can be replaced by the duration of the k-th pulse. By defining $\mathrm{p}_{\mathrm{k}}=\mathrm{Q}_{\mathrm{k}} / \sum_{\mathrm{n}=1}^{\mathrm{N}} \mathrm{Q}_{\mathrm{n}}$, we have found that the variance $\kappa_{1}=\left\langle\chi^{2}\right\rangle-\left\langle\chi^{2}\right.$, where $\langle\mathrm{f}(\chi)\rangle=\sum_{\mathrm{n}=1}^{\mathrm{N}} \mathrm{p}_{\mathrm{k}} \mathrm{f}\left(\chi_{\mathrm{k}}\right)$, of the natural time $\chi$ with respect to the distribution $\mathrm{p}_{\mathrm{k}}$ may be used for identifying criticality, and hence the SES activities. More specifically, the following relation should hold for SES activities

$\kappa_{1} \approx 0.070$ 
Beyond the condition of Eq. (1), we have shown that the SES activities, when analyzed in natural time, exhibit infinitely ranged temporal correlations and obey the conditions (Varotsos et al., 2005, 2006a,b):

$\mathrm{S}, \mathrm{S}_{-}<\mathrm{S}_{\mathrm{u}}$

where $\mathrm{S}$ is the entropy $\mathrm{S}$ in natural time defined as: $\mathrm{S} \equiv<\chi \ln \chi>-$ $<\chi>\ln \langle\chi\rangle$ (Varotsos et al., 2003a) and $S_{-}$is the entropy obtained upon time reversal. Eq. (2) states that both $S_{\text {and }} S_{-}$are smaller than the value $S_{u}$ $(=\ln 2 / 2-1 / 4 \approx 0.0966)$ of a "uniform" (u) distribution, e.g. when all $\mathrm{p}_{\mathrm{k}}$ are equal.

The fact that SES activities exhibit critical dynamics, is believed to be related to their generation mechanism (see Varotsos et al., 1993, and references therein). In the focal area of an impending earthquake (EQ hereafter), which contains ionic materials, the stress gradually increases. In ionic solids a number of extrinsic defects are always formed because they contain aliovalent impurities. These extrinsic defects are attracted by the nearby impurities and hence form electric dipoles the orientation of which can change through defect migration. When the stress (pressure) $\sigma$ reaches a critical value $\sigma_{\mathrm{cr}}$, a cooperative orientation of these dipoles occurs generating SES

We now summarize the detrended fluctuation analysis DFA (Peng et al., 1994; Taqqu et al., 1995) which is a novel method that has been developed to address the problem of accurately quantifying long range correlations in non-stationary fluctuating signals. It has been applied to diverse fields ranging from DNA (Peng et al., 1993; Stanley et al., 1999), to meteorology (Ivanova and Ausloos, 1999), and economics (Vandewalle and Ausloos, 1997; Ivanov et al., 2004). DFA is, in short, a modified root-mean-square (rms) analysis of a random walk. In principle, it estimates the deviations from the local trends $y_{s}(n)$ of a non-stationary long time series of length $\mathrm{N}$ piecewise by dividing it into small segments with length $s$ and compute the Fluctuation function $F(s)$, which is the variance of $y_{s}(n)$ :

$\mathrm{F}(\mathrm{s})=\sqrt{\frac{1}{N} \sum_{n=1}^{N}\left[y_{s}(n)\right]^{2}}$

$\mathrm{F}(\mathrm{s})$ corresponds to the trend-eliminated root mean square displacement of the random walker. Then, the above computation is repeated for a broad number of scales $s$ to provide a relationship between $F(s)$ and $s$.

When a power-law relation between $\mathrm{F}(\mathrm{s})$ and s, i.e.

$\mathrm{F}(\mathrm{s}) \propto \mathrm{s}^{\alpha}$

is found, it indicates the presence of scaling-invariant (fractal) behavior embedded in the fluctuations of the signal (Peng et al., 1994; Taqqu et al., 1995). The fluctuations can be characterized by the scaling exponent $\alpha$, a self-similarity parameter: If $\alpha=0.5$, there are no correlations in the data and the signal is uncorrelated (white noise); the case $\alpha<0.5$ corresponds to anti-correlations, meaning that large values are most likely to be followed by small values and vice versa. If $\alpha>0.5$, there are long-range correlations, which are stronger for higher $\alpha$ (Bashan et al., 2008). Note that $\alpha>1$ indicates a non-stationary local average of the data and the value $\alpha=1.5$ indicates Brownian motion (integrated white noise).

For stationary signals with long-range power-law correlations the value of the scaling exponent $\alpha$ is interconnected with the exponent $\beta$ characterizing the power spectrum $\mathrm{S}(\mathrm{f}) \sim \mathrm{f}^{-\beta}$ (f=frequency) through (Peng et al., 1993)

$\beta=2 \alpha-1$

When employing natural time, DFA seems to distinguish (Varotsos et al., 2003b) SES activities from artificial noise because, for the SES activities the $\alpha$-values lie approximately in the range

$0.9 \leq \alpha \leq 1.0$ while for the artificial noise (caused by man-made sources) investigated in Greece (Varotsos et al., 2003a,b) the $\alpha$-values are markedly smaller, i.e., $\alpha=0.65-0.8$. In other words, the artificial noise recorded in Greece, which at the most lasts for $24 \mathrm{~h}$, may have long-range correlations, e.g. $\alpha \approx 0.75$ (see Fig. 9 of Varotsos et al. (2003a)), but none of several artificial noises studied was found to exhibit infinitely ranged longrange correlations (i.e., having $\alpha$-value close to unity).

\section{Data analysis and results}

Let us suppose that we have a long time series of data s(i) (shown in red in the example of Fig. 1), with a duration appreciably larger than $24 \mathrm{~h}$ for instance, and we are forced to remove the same segment of these daily data. The portion of the 24 hour data that remain will be hereafter labeled $\mathrm{p}_{\mathrm{r}}$ and the number of data corresponding to one period, say $24 \mathrm{~h}$, T. Thus, every T samples, $\left(1-\mathrm{p}_{\mathrm{r}}\right) \mathrm{T}$ of them (belonging to the shaded parts of Fig. 1) are removed. The remaining segments (blue in Fig. 1) are concatenated to form the new time series c(i) which is subsequently read in natural time. We now impose the following conditions (7) and (8) on c(i) for classifying the signal as SES activity. The condition (7) comes from the relation (6) after considering the reasonable experimental error:

$0.85 \leq \alpha \leq 1.10$

The condition (8) comes from Eqs. (1) and (2) also by considering the reasonable experimental error in $\kappa_{1}$ :

$\left|\kappa_{1}-0.07\right| \leq 0.01, S \leq S_{u}, S_{-} \leq S_{u}$

In the following subsections, in order to solve our problem, synthetic signals will be produced and analyzed whether they obey conditions (7) and/or (8) using a Monte Carlo comprising $10^{3}$ realizations. The Monte Carlo procedure has been used to "average" over the possible realizations of the synthetic SES activities and noises that will be discussed later in Sections 2.1 and 2.2 as well as the fact that both types of electric signals may start any time of the day. Thus, one should randomly select an integer $\mathrm{i}_{\text {init }}$ from 1 up to $\mathrm{T}$, and keep in $\mathrm{c}(\mathrm{i})$ the samples $\mathrm{s}\left(\mathrm{i}_{\text {init }}\right)$ to $\mathrm{s}\left(\mathrm{i}_{\text {init }}+\mathrm{p}_{\mathrm{r}} \mathrm{T}-1\right)$ of $\mathrm{s}(\mathrm{i})$, i.e., we keep $\mathrm{p}_{\mathrm{r}} \mathrm{T}$ samples in total. The next segment to be kept in $\mathrm{c}(\mathrm{i})$ is $\left(1-\mathrm{p}_{\mathrm{r}}\right) \mathrm{T}$ samples after $\mathrm{s}\left(\mathrm{i}_{\text {init }}+\mathrm{p}_{\mathrm{r}} \mathrm{T}-1\right)$, starting from $\mathrm{s}\left[\mathrm{i}_{\text {init }}+\mathrm{p}_{\mathrm{r}} \mathrm{T}-1+\right.$ $\left(1-\mathrm{p}_{\mathrm{r}}\right) \mathrm{T}+1=\mathrm{i}_{\text {init }}+\mathrm{T}$ ] up to $\mathrm{s}\left(\mathrm{i}_{\text {init }}+\mathrm{T}+\mathrm{p}_{\mathrm{r}} \mathrm{T}-1\right)$ and so on (see the blue lines in Fig. 1). This way we periodically remove $\left(1-p_{r}\right) T$ samples and keep $\mathrm{p}_{\mathrm{r}} \mathrm{T}$ every $\mathrm{T}$ samples from the original signal $\mathrm{s}(\mathrm{i})$. This Monte Carlo simulation allows us to evaluate the probability to identify the original signal as a SES activity.

The probability that the condition (7) is satisfied will be hereafter labeled $\mathrm{p}_{1}$. By the same token, the probability to satisfy the condition (8) is designated by $\mathrm{p}_{2}$. Finally, the probability to obey either condition (7) or condition (8) will be labeled $\mathrm{p}_{3}$. Upon considering the number of the Monte Carlo realizations $\left(\mathrm{M}=10^{3}\right)$, a plausible estimation error

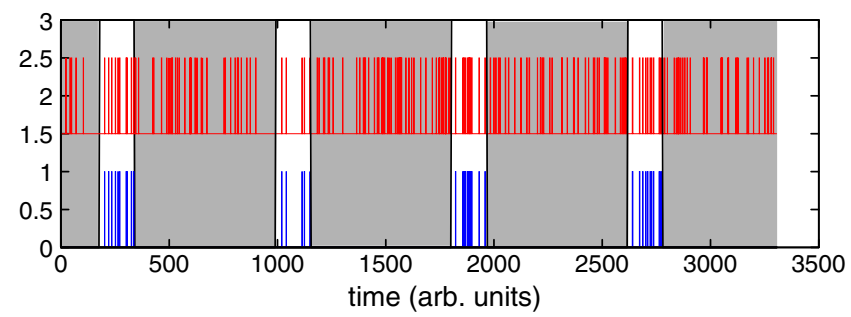

Fig. 1. An example of the procedure described in Section 2.1, showing data segments that are periodically removed every $\mathrm{T} \approx 813$ arbitrary units from the original dichotomous time-series (red). The gray shaded areas correspond to the high noise periods (e.g. 06:0022:00 LT in Japan) which have to be discarded daily. 
(3STD $/ \sqrt{ } \mathrm{M}$ ) at the most around $5 \%$ is expected (cf. $1 / \sqrt{ } 10^{3} \approx 0.032$, and STD stands for the standard deviation of the quantity calculated by Monte Carlo, e.g., see Weinzierl, 2000).

Since we are interested in the low cultural noise night-window, we hereafter focus on the $p_{r}$ values varying from $p_{r}=0.2$ to roughly $\mathrm{p}_{\mathrm{r}}=0.3$. The following two subsections summarize the results obtained for synthetic long duration SES activities and synthetic noise, respectively. The first subsection concludes that when using $\mathrm{p}_{3}$ the remaining data are highly probable to enable the identification of a SES activity. This means that the use of either condition (7) or conditions (8), i.e. $p_{3}$, is better to be used for the classification of a signal as a SES activity. Thus, such a probability can be thought as the conditional probability $\mathrm{p}$ (SES| SES), i.e., the probability to identify a true SES activity as such, after periodic data loss (cf. the complementary probability to miss a true SES is labeled $\left.\mathrm{p}_{\text {miss }} \equiv 1-\mathrm{p}(\mathrm{SES} \mid \mathrm{SES})\right)$. The second subsection deals with another conditional probability $\mathrm{p}$ (SES|noise), labeled pnoise (Fig. 2), to erroneously identify a noise as SES activity when using conditions (7) and (8). Finally, results from a real world data set are provided in the third subsection.

\subsection{Results obtained from synthetic long duration SES activities}

In order to construct synthetic long duration SES activities, one has to make use of a model for both the time-series of the pulse durations $Q_{k}$ and the appropriate waiting times $\mathrm{W}_{\mathrm{k}}$, i.e., the times elapsed between consecutive emissions of SES pulses. Since for actual data (e.g. see Varotsos et al., 2003b) $Q_{k}$ and $W_{k}$ exhibit different behavior when studied by DFA, we follow below different methods to generate the corresponding time series.: We start with the results of the DFA of the pulse durations $\mathrm{Q}_{\mathrm{k}}$ : Its DFA analysis reveals (Varotsos et al., 2003b) an exponent $\alpha \approx 1$, i.e., $\mathrm{Q}_{\mathrm{k}}$ exhibit very strong long-range correlations (infinitely ranged long-range correlations). This led Varotsos et al. (2006b) to study in natural time the one sided segments of a fractional Gaussian noise ( $\mathrm{fGn}$ ). $\mathrm{fGn}$, which is stationary and Gaussian with zero mean, corresponds to the time series of the increments of a fractional Brownian motion ( $\mathrm{fBm}$ ) $\mathrm{B}_{\mathrm{H}}(\mathrm{t})$ with self-similarity index $\mathrm{H}$ (Mandelbrot and van Ness, 1968); the latter is the Gaussian generalization of the Brownian motion - random walk - in the sense that its root mean square displacement is proportional to the $\mathrm{H}$-th power of the time lag, i.e., $\sqrt{\left\langle\left(B_{H}(t)-B_{H}(s)\right)^{2}\right\rangle} \propto|t-s|^{H}$ (cf. for $\mathrm{H}=0.5$ we simply recover the behavior of a random walk). Thus, for $\mathrm{H}=0.5 \mathrm{fGn}$ is just white noise whereas for $\mathrm{H}>0.5$ it corresponds to DFA exponents $\alpha \approx \mathrm{H}$. Here, we model the durations $Q_{k}$ as the one sided segments of a fractional Gaussian noise with $H \approx 1$, because Varotsos et al. (2006b) have shown that in this case both conditions (7) and (8) are satisfied. We now turn to the time series of the waiting times $\mathrm{W}_{\mathrm{k}}$ and consider that for actual data their DFA exponent $\alpha$ scatters around $\alpha \approx 0.5$ (e.g. see Table III of Varotsos et al., 2003b), which reflects that there are almost no temporal correlations or at least the absence of significant long-range time correlations. Additionally, in the Supplemental material of Varotsos et al. (2003a), we showed that the waiting times' statistics cannot be fully accounted for by an exponential distribution, thus they cannot be fully characterized as simply random (Poissonian). Moreover, their distributions for various SES activities (e.g. see Table II of the Supplemental material of Varotsos et al., 2003a) do not seem to share obviously common properties. This may be understood in the frame of the aforementioned SES generation mechanism (see Varotsos et al., 1993, and references therein) during the preparation stage of a strong EQ: A

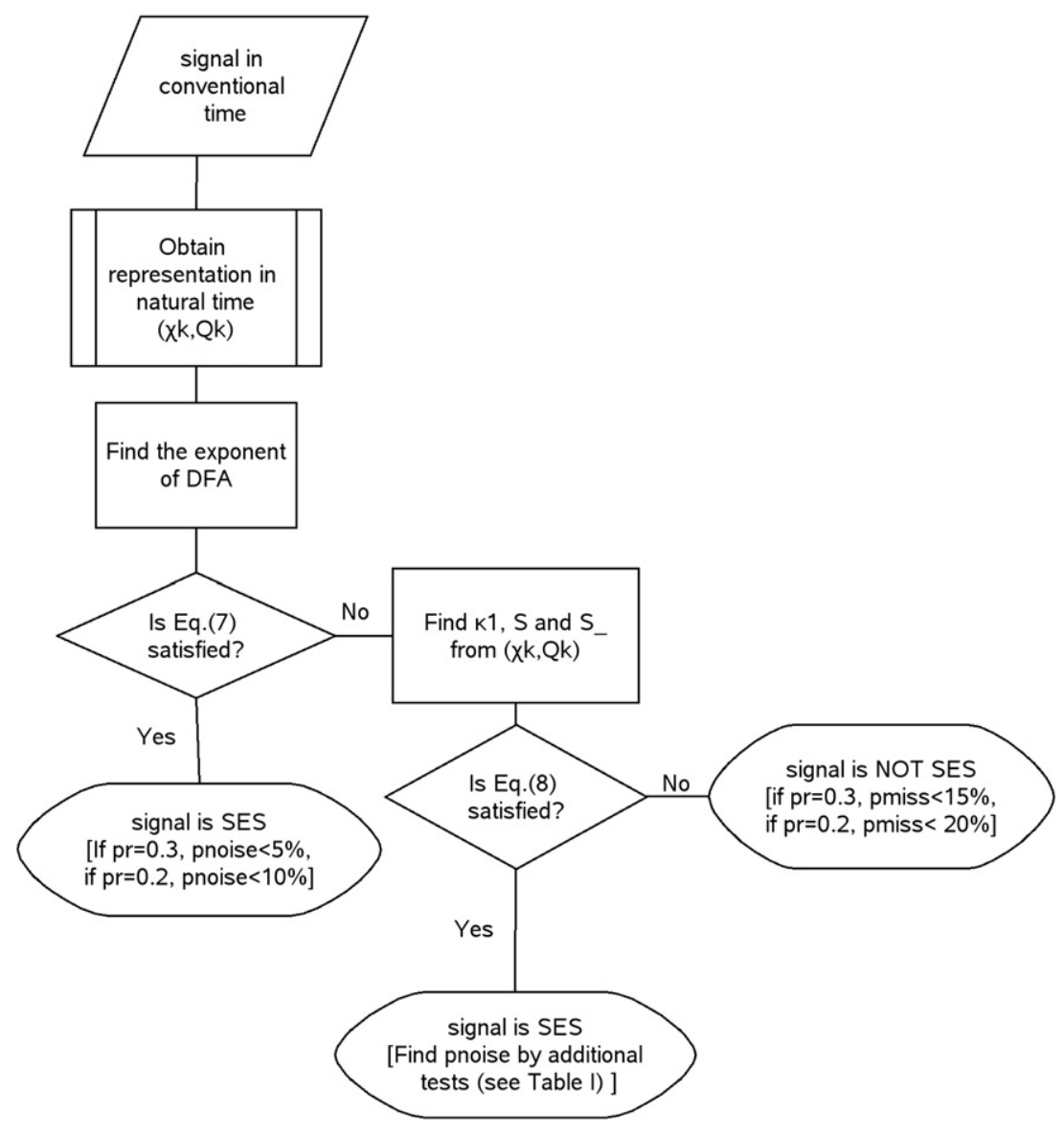

Fig. 2. Flow-chart of the method proposed. 
simultaneous achievement of the stimulating (critical) stress $\sigma_{\mathrm{cr}}$ at all points in the stressed EQ preparation volume is not intuitively expected (Varotsos and Lazaridou, 1991; see also p.258 in Varotsos, 2005). When the condition $\sigma=\sigma_{\mathrm{cr}}$ is fulfilled in a sub-volume of the EQ preparation volume, a SES pulse is emitted. Thus, when this occurs consecutively in various sub-volumes, a series of SES pulses is emitted which constitutes a SES activity. In other words, the "points" obeying the condition $\sigma=\sigma_{\mathrm{cr}}$ should lie on a "surface" A, let us call it critical stress front, which may be very complicated in view of the existing inhomogeneities. This front sweeps through the preparatory volume with highly stochastic (stickslip like) movement, the details of which (e.g., the "stick" intervals) determine the waiting times $\mathrm{W}_{\mathrm{k}}$ and may depend on the specific preparation zone and the time to the mainshock. Such a complex behavior is difficult to model, but in the following we will assume as first approximation that the waiting times $\mathrm{W}_{\mathrm{k}}$ between consecutive SES pulses are independent and identically distributed (iid) random variables from an exponential distribution (in simple words, this means that $\mathrm{W}_{\mathrm{k}}$ come from an unbiased - ideal - exponential random number generator). This assumption will be also applied to the case of $\mathrm{W}_{\mathrm{k}}$ for the synthetic noise treated in the next subsection.

Along these lines, we generate synthetic dichotomous signals $d(t)$ that resemble long duration SES activities by the procedure described in detail below:

(i) We first generate the durations of the pulses $T_{o n}(k) \equiv Q_{k}, k=0,1$, $2, \ldots, N$ with $\mathrm{N} \geq 200$, that come from the one sided segments of a fractional Gaussian noise with $\mathrm{H}=1$ and satisfy both conditions (7) and (8) (cf. the method to produce such segments has been described in detail by Varotsos et al., 2006b, and can be also visualized in Fig. 5 of the Supplemental material of that reference).

(ii) We then generate the waiting times between consecutive pulses, i.e., $\mathrm{T}_{\text {off }}(\mathrm{k}) \equiv \mathrm{W}_{\mathrm{k}}$, which are independent exponentially distributed random variables with average value $\tau_{\text {off }}=\tau_{\text {on }} / \lambda$, where $\tau_{\text {on }}$ is the average duration of pulses (i.e., $\tau_{\text {on }} \equiv<\mathrm{T}_{\text {on }}(\mathrm{k})>$ ) and $\lambda$ an arbitrary dimensionless parameter corresponding to the ratio of the probabilities to find the system active over the probability to find the system inactive. Since we investigate the case of long duration SES activities, the aforementioned critical stress front $A$, obeying the condition $\sigma=\sigma_{\mathrm{cr}}$, is naturally expected to propagate "slowly". This should correspond to long "stick" intervals $\mathrm{W}_{\mathrm{k}}$ and hence increased probability to find the system inactive. For these reasons, a typical value corresponding to ten times higher probability to find the system inactive than active, i.e., $\lambda=0.1$, has been considered.

(iii) We then construct $d(t)$ by assigning to it the value of 1 if the system is active, i.e., during the emission of a pulse, at time $t$, or 0 otherwise. Namely, we have $d(t)=0$ for $t<T_{\text {off }}(0), d(t)=1$ for $\mathrm{t} \in\left[\mathrm{T}_{\text {off }}(0), \mathrm{T}_{\text {off }}(0)+\mathrm{T}_{\text {on }}(1)\right]$, then $\mathrm{d}(\mathrm{t})=0$ again for $\mathrm{t} \in\left(\mathrm{T}_{\text {off }}(0)+\right.$ $\left.\mathrm{T}_{\text {on }}(1), \mathrm{T}_{\text {off }}(0)+\mathrm{T}_{\text {on }}(1)+\mathrm{T}_{\text {off }}(1)\right)$ and $\mathrm{d}(\mathrm{t})=1$ for $\mathrm{t} \in\left[\mathrm{T}_{\text {off }}(0)+\right.$ $\left.\mathrm{T}_{\text {on }}(1)+\mathrm{T}_{\text {off }}(1), \mathrm{T}_{\text {off }}(0)+\mathrm{T}_{\text {on }}(1)+\mathrm{T}_{\text {off }}(1)+\mathrm{T}_{\text {on }}(2)\right]$ and so on.

The time-series $d(t)$ generated this way was then periodically cut, following the Monte Carlo procedure described in Section 2, where the " 24 hours" period has been selected by $\mathrm{T}=\mu\left(\tau_{\text {off }}+\tau_{\text {on }}\right)$, and $\mu$ is another dimensionless parameter corresponding to the average number of pulses emitted during the 24 hour period. Note that in order to make a reliable DFA analysis we should have at least around ten pulses (see p.301 in Varotsos, 2005, and p.3 of Ma et al., 2010) in each remaining data segment every "day". This reflects that even upon $80 \%$ data loss, ten pulses should still remain every "day", thus hereafter we select the value $\mu=50$. Obviously, if we consider a larger $\mu$ value, e.g. $\mu=100$ or 200 , our results will become better. For $\mathrm{p}_{\mathrm{r}}=0.2, \lambda=0.1$ and $\mu=50$, we obtain $p_{1}=0.45, p_{2}=0.71$ and $p_{3}=0.83$ (with a plausible estimation error $5 \%$ ). These values change to $\mathrm{p}_{1}=0.57, \mathrm{p}_{2}=0.73$ and $\mathrm{p}_{3}=0.87$ when $\mathrm{p}_{\mathrm{r}}$ increases to 0.3 (see Table 1 ).

\subsection{Results from synthetic noise}

Here, we discuss cases of uncorrelated (in natural time) noise which gives rise to dichotomous signals, e.g. random telegraph signals (Varotsos, 2005), which may be confused with SES activities. In particular, the following three types of synthetic noise will be discussed: (a) Markovian dichotomous time-series, (b) a noise signal in which the durations of activity come from a uniform distribution (i.e., $Q_{k}$ are independent random variables uniformly distributed in $\left[0,2 \tau_{\text {on }}\right]$ ) or (c) from a Gaussian distribution (i.e., $\mathrm{Q}_{\mathrm{k}}$ are iid originating from a Gaussian distribution with an average duration $\tau_{\text {on }}$ and a standard deviation $\sigma=\beta \tau_{\text {on }}$, where $\beta$ is a dimensionless constant necessarily small (e.g., $\beta=0.1$ ) to ensure the positivity of the "durations" $Q_{k}$ ).

(a) Markovian dichotomous signals. This is the case of the Random Telegraph Signals (RTS) in which both the durations of activity and inactivity are iid exponential random variables with average durations $\tau_{\text {on }}$ and $\tau_{\text {off, }}$, respectively. For the case $\mathrm{p}_{\mathrm{r}}=0.2, \lambda=0.1$ and $\mu=50$, we obtain $\mathrm{p}_{1}=0.08, \mathrm{p}_{2}=0.43$ and $\mathrm{p}_{3}=0.47$. These values change to $\mathrm{p}_{1}=0.02, \mathrm{p}_{2}=0.43$ and $\mathrm{p}_{3}=0.44$ when $\mathrm{p}_{\mathrm{r}}$ becomes 0.3 (Table 1 ). Hence, we observe that when using either $\mathrm{p}_{2}$ or $\mathrm{p}_{3}$ it is probable that a Markovian dichotomous signal may be misinterpreted as SES activity. To avoid it, we have to perform an additional test described in Appendix 1.

(b) Noise signals with uniformly distributed $T_{\text {on }}$. When the durations $\mathrm{T}_{\mathrm{on}}(\mathrm{k})\left(\equiv \mathrm{Q}_{\mathrm{k}}\right)$ are independent and identically distributed uniform random variables in $\left[0,2 \tau_{\text {on }}\right]$, the results for $\mathrm{p}_{\mathrm{r}}=0.2, \lambda=0.1$ and $\mu=50$ are $p_{1}=0.07, p_{2}=0.33$ and $p_{3}=0.37$. These values change to $\mathrm{p}_{1}=0.03, \mathrm{p}_{2}=0.29$ and $\mathrm{p}_{3}=0.31$ when $\mathrm{p}_{\mathrm{r}}$ becomes 0.3 . Hence, we again observe that when using either conditions (7) or (8) (i.e., the case of $p_{3}$ ) for the identification of a SES activity, it is probable to misinterpret such a noise as SES. To avoid it, we have to perform an additional test described in Appendix 2.

(c) Noise signals with Gaussian distributed $T_{o n}$. In such a case for $\beta=0.1$ and $\mathrm{p}_{\mathrm{r}}=0.2, \lambda=0.1$ and $\mu=50$, we obtain $\mathrm{p}_{1}=0.08$, $\mathrm{p}_{2}=0.02$ and $\mathrm{p}_{3}=0.10$. These values change to $\mathrm{p}_{1}=0.01$, $\mathrm{p}_{2}=0.00$ and $\mathrm{p}_{3}=0.02$ when $\mathrm{p}_{\mathrm{r}}$ becomes 0.3 . These values suggest that it is improbable to misinterpret such a noise as SES when using $\mathrm{p}_{2}$ either for $\mathrm{p}_{\mathrm{r}}=0.2$ or $\mathrm{p}_{\mathrm{r}}=0.3$. The values of $\mathrm{p}_{1}$ and $\mathrm{p}_{3}$ alone also achieve a satisfactory distinction between noise and SES activities but to a lesser extent than $\mathrm{p}_{2}$ (see Table 1 ).

\subsection{Results from a real world dataset}

SES activities of appreciably long duration around a few weeks or more, i.e., similar to the one observed by Uyeda et al. $(2002,2009)$ almost 2 months before the case of Izu island swarm in Japan, have not been recorded in Greece. Here, we consider as an example the SES

Table 1

The values of $\mathrm{p}_{1}, \mathrm{p}_{2}$ and $\mathrm{p}_{3}$ (with an estimation error $5 \%$ ) obtained with $\lambda=0.1$ and $\mu=50$ for the synthetic SES activities (SES), Markovian dichotomous signals (Markovian), noise signals with uniformly distributed $\mathrm{T}_{\mathrm{on}}$ (Uniform) and noise signals with Gaussian distributed $\mathrm{T}_{\text {on }}$ with $\beta=0.1$ (Gaussian). The two cases $\mathrm{p}_{\mathrm{r}}=0.2$ or $\mathrm{p}_{\mathrm{r}}=0.3$ are shown.

\begin{tabular}{llccc}
\hline Type of signal & $\mathrm{p}_{\mathrm{r}}$ & $\mathrm{p}_{1}(\%)$ & $\mathrm{p}_{2}(\%)$ & $\mathrm{p}_{3}(\%)$ \\
\hline SES $_{\text {Markovian }}{ }^{\mathrm{a}}$ & 0.2 & 45 & 71 & 83 \\
Uniform $^{\mathrm{b}}$ & 0.2 & 8 & 43 & 47 \\
Gaussian $_{\text {SES }}$ & 0.2 & 7 & 33 & 37 \\
Markovian $^{\mathrm{a}}$ & 0.2 & 8 & 2 & 10 \\
Uniform $^{\mathrm{b}}$ & 0.3 & 57 & 73 & 87 \\
Gaussian & 0.3 & 2 & 43 & 44 \\
\hline
\end{tabular}

a Secure distinction from SES is achieved by the statistics of $\sigma\left(T_{o n}\right) / \mu\left(T_{o n}\right)$, see Appendix 1.

b Secure distinction from SES is achieved by the statistics of $\rho=\left[\left(T_{\text {on }}\right)_{\max }-\left(T_{\text {on }}\right)_{\min }\right] /$ $\sigma\left(\mathrm{T}_{\mathrm{on}}\right)$, see Appendix 2. 
activity that preceded (Varotsos et al. (2009)) the most recent major EQ in Greece: This, which had almost 1.5 day duration, lasted from February 29 to March 2, 2008, was followed by a magnitude Mw6.4 EQ at $38.0^{\circ} \mathrm{N}$ $21.5^{\circ} \mathrm{E}$ on June 8,2008 . Its original time series, which is not of obvious dichotomous nature, is reproduced in Fig. 3(a). We now attempt to answer the following question: If such a SES activity would have been recorded in Japan, could its identification become possible by employing the procedure proposed here?

Before proceeding we note that the identification of this SES activity in Greece has been described in detail by Varotsos et al. (2009). Chief among the results obtained are the following: first, the signal as it is evident from an inspection of Fig. 3(a), comprising a number of pulses, is superimposed on a background which exhibits frequent magnetotelluric (MT) variations. After subtracting these MT variations (Varotsos et al., 2009), we find the signal depicted in Fig. 3(b), which provides the time series that should be considered for further analysis. Its analysis in natural time leads to the representation depicted in Fig. 4 and the parameters $\kappa_{1}, S$ and $S_{-}$resulted in its identification as SES activity.

In order to answer the question on the possibility to identify this almost one and a half day long - SES activity after significant data loss and since it may have started any time of the day, a Monte Carlo calculation similar to that described in Section 2 was employed. In particular, we randomly select the first segment to keep starting at some time $t_{0}$ uniformly distributed during the first $24 \mathrm{~h}$ (i.e., the first 86,400 samples since the sampling rate is 1 sample/s), then we discard the experimental data until $t_{0}+24 \mathrm{~h}$, when the second segment to keep starts - of course, depending on $t_{0}$ the available SES data may not be enough to allow the selection of a second segment. This is repeated for various values of $t_{0}$ and the Monte Carlo simulation shows that when removing $70 \%$ of the data (i.e., $\mathrm{p}_{\mathrm{r}}=0.3$ ), we find a probability of $\approx 67 \%$ to correctly identify this SES activity $\left(\mathrm{p}_{1}=0.40, \mathrm{p}_{2}=0.54\right.$ and $\left.\mathrm{p}_{3}=0.67\right)$. This probability becomes somewhat smaller, i.e., $\approx 62 \%$, upon increasing the data loss to $80 \%\left(p_{1}=0.41, p_{2}=0.40\right.$ and $\left.p_{3}=0.62\right)$. These values of the probability are expected to become markedly larger if the duration of the SES activity were similar to the one observed before the 2000 Izu Island seismic swarm in Japan.

\section{Summary of results and conclusion}

A flow diagram of the method proposed here to identify in the remaining data SES activities as well as to avoid misinterpreting them as noise, is given in Fig. 2. It reveals that after reading the remaining data in natural time, the DFA exponent $\alpha$ may be enough to achieve that

(a)

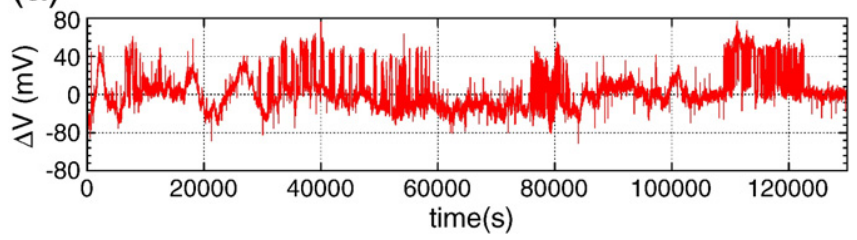

(b)

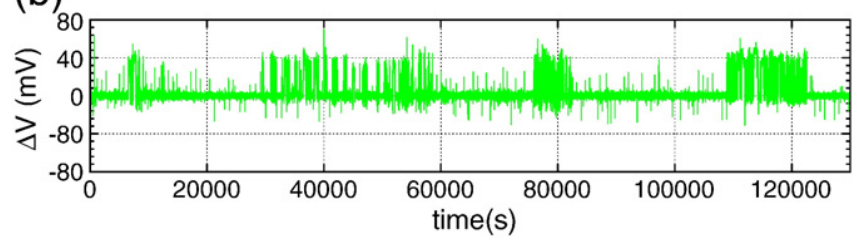

Fig. 3. The SES activity from February 29 to March 2, 2008: (a) original time-series (b) the same as (a) but after subtracting the MT background variations by applying the procedure described in (Varotsos et al., 2009).

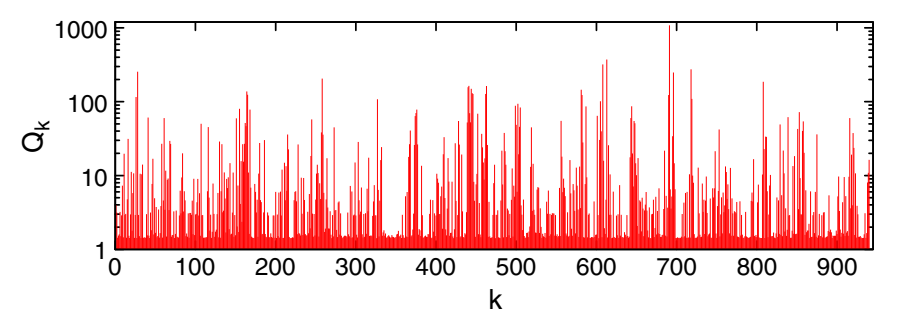

Fig. 4. Natural time representation of the signal depicted in Fig. 3(b) according to Fig. 7 of Varotsos et al. (2009). The "durations" $Q_{k}$ are here presented in arbitrary units.

purpose; otherwise, the quantities $\kappa_{1}, \mathrm{~S}$, and $\mathrm{S}_{-}$as well the statistics of the duration of pulses should be also studied.

Our main conclusion states that when employing two modern techniques, i.e., natural time analysis and DFA, a distinction between SES activities (critical dynamics) and artificial noise becomes possible even after removing periodically a significant portion of the data. In particular, when using synthetic long duration SES activities, upon removing $70 \%$ of the data (e.g., the data from 06:00 to 22:00 LT like in Japan), we have a probability around $87 \%$, or larger, to identify correctly a SES activity. This probability becomes somewhat smaller, i.e., 83\%, when the data removal increases to $80 \%$. In addition, when investigating a real world data set referring to a SES activity of just one and a half day duration, the following results are obtained: upon removing $70 \%$ (80\%) of the data, we find a probability of $\approx 67 \%(62 \%)$ to correctly identify this SES activity. The probability is expected to increase significantly for SES activities of longer duration, i.e., a few weeks or more as in the case of the Izu Island seismic swarm.

\section{Appendix 1. An additional test to discriminate Markovian dichotomous signals from SES activities}

Since Markovian dichotomous signals are probable to be misinterpreted as SES activities because their $\mathrm{p}_{3}$ values are close to $50 \%$, we additionally check the ratio $\sigma\left(\mathrm{T}_{\text {on }}\right) / \tau_{\text {on }}$ of the standard deviation versus the average duration of the pulses: The Markovian dichotomous signals have a ratio varying (even in the case of 50 pulses) in the range [0.72, 1.34] with probability $98 \%$. For the SES model used in Section 2.1, the probability to have a ratio larger than 0.70 is only $0.2 \%$. In other words, we should compare the $\sigma\left(\mathrm{T}_{\mathrm{on}}\right) / \tau_{\mathrm{on}}$-value of the signal under investigation with the corresponding value for an exponential distribution having a number of samples equal to the number of pulses in the signal. Thus, when using either conditions (7) or (8) (i.e., the case of $\mathrm{p}_{3}$ ) for the identification of a SES activity, we should also study the statistics of the durations of the recorded pulses to avoid misinterpreting a Markovian dichotomous signals as SES.

\section{Appendix 2. An additional test to discriminate noise signals with uniformly distributed $T_{\text {on }}$ from SES activities}

As mentioned in Section 2.2(b), $\mathrm{p}_{3}$ in this case may reach values around 33\%. In order to avoid misinterpreting such a signal as SES activity, we again (see Appendix 1) resort to the statistics of the durations of the pulses. In particular, we consider the ratio $\rho$ of the range of durations for such a noise over their standard deviation, i.e., $\left[\left(\mathrm{T}_{\mathrm{on}}\right)_{\max }-\left(\mathrm{T}_{\mathrm{on}}\right)_{\min }\right] /$ $\sigma\left(\mathrm{T}_{\mathrm{on}}\right)(\equiv \rho)$, which varies (even in the case of 50 pulses) in the range $[3.0,3.8]$ with probability $95 \%$. Moreover, the probability $\mathrm{P}(\rho<3.7)$ that $\rho$ is smaller than 3.7 is around $94 \%$. On the other hand, the probability that a SES activity, modeled according to Section 2.1, has a $\rho$-value larger than 3.8 is about $87.5 \%$ and increases to $92 \%$ when we consider a $\rho$ value larger than 3.7. Thus, when using either conditions (7) or (8) (i.e., the case of $\mathrm{p}_{3}$ ) for the identification of a SES activity, we should also study the statistics of the durations of the recorded pulses to obtain $\rho$. Then there is approximately $90 \%$ probability to safely distinguish SES from a noise signal with $\mathrm{T}_{\text {on }}$ uniformly distributed even in the extreme case of 
recording only 50 pulses. This result becomes better if 100 pulses are recorded in the noise-free segments. In this case, $\rho$ varies in the range $[3.1,3.8]$ with probability $98 \%$, and $\mathrm{P}(\rho<3.7)$ is $96 \%$; on the other hand, the synthetic SES activities treated in Section 2.1 result in a small $\mathrm{P}$ $(\rho<3.7)$ value $\sim 3 \%$.

\section{References}

Bashan, A., Bartsh, R., Kantelhardt, J.W., Havlin, S., 2008. Comparison of detrending methods for fluctuation analysis. Physica A 387, 5080-5090.

Ivanov, P.C., Yuen, A., Podobnik, B., Lee, Y., 2004. Common scaling patterns in intertrade times of U.S. stocks. Phys. Rev. E 69, 056107.

Ivanova, K., Ausloos, M., 1999. Application of the detrended fluctuation analysis (DFA) method for describing cloud breaking. Phys. A 274, 349-354.

Ma, Q.D.Y. Bartsch, R.P., Bernaola-Galván, P. Yoneyama, M. Ivanov, P.C. 2010. Effect of extreme data loss on long-range correlated and anti-correlated signals quantified by detrended fluctuation analysis. Phys. Rev. E 81, 031101.

Mandelbrot, B.B., van Ness, J.W., 1968. Fractional noises and applications. SIAM Rev. 10, 422-437.

Orihara, Y., Kamogawa, M., Nagao, T., Uyeda, S., 2009. Independent component analysis of geoelectric field data in the northern Nagano, Japan. Proc. Jpn Acad. Ser. B Phys. Biol. Sci. 85, 435-442.

Peng, C.K., Buldyrev, S.V., Goldberger, A.L., Havlin, S., Simons, M., Stanley, H.E., 1993 Finite-size effects on long-range correlations: implications for analyzing DNA sequences. Phys. Rev. E 47, 3730-3733.

Peng, C.K., Buldyrev, S.V., Havlin, S., Simons, M., Stanley, H.E., Goldberger, A.L., 1994. Mosaic organization of dna nucleotides. Phys. Rev. E 49, 1685-1689.

Skordas, E.S., Sarlis, N.V., Varotsos, P.A., 2010. Effect of significant data loss on identifying electric signals that precede rupture by detrended fluctuation analysis in natural time. Chaos 20, 033111.

Stanley, H.E., Buldyrev, S.V., Goldberger, A.L., Havlin, S., Peng, C.K., Simon, M., 1999. Scaling features of noncoding DNA. Phys. A 273, 1-18.

Taqqu, M.S., Teverovsky, V., Willinger, W., 1995. Estimators for long-range dependence: an empirical study. Fractals 3, 785-798.

Uyeda, S., Nagao, T., Orihara, Y., Yamaguchi, T., Takahashi, I., 2000. Geoelectric potential changes: possible precursors to earthquakes in Japan. Proc. Natl. Acad. Sci. USA 97, 4561-4566.
Uyeda, S., Hayakawa, M., Nagao, T., Molchanov, O., Hattori, K., Orihara, Y., Gotoh, K, Akinaga, Y., Tanaka, H., 2002. Electric and magnetic phenomena observed before the volcano-seismic activity in 2000 in the Izu island region, Japan. Proc. Natl. Acad. Sci. USA 99, 7352-7355.

Uyeda, S., Kamogawa, M., Tanaka, H., 2009. Analysis of electrical activity and seismicity in the natural time domain for the volcanic-seismic swarm activity in 2000 in the Izu island region, Japan. J. Geophys. Res. 114, B02310.

Vandewalle, N., Ausloos, M., 1997. Coherent and random sequences in financial fluctuations. Phys. A 246, 454-459.

Varotsos, P., 2005. The Physics of Seismic Electric Signals. TERRAPUB, Tokyo.

Varotsos, P., Alexopoulos, K., 1984a. Physical properties of the variations of the electric field of the earth preceding earthquakes, I. Tectonophysics 110, 73-98.

Varotsos, P., Alexopoulos, K., 1984b. Physical properties of the variations of the electric field of the earth preceding earthquakes, II. Tectonophysics 110, 99-125.

Varotsos, P., Lazaridou, M., 1991. Latest aspects of earthquake prediction in Greece based on seismic electric signals. Tectonophysics 188, 321-347.

Varotsos, P., Alexopoulos, K., Lazaridou, M., 1993. Latest aspects of earthquake prediction in Greece based on seismic electric signals, II. Tectonophysics 224, 1-37.

Varotsos, P.A., Sarlis, N.V., Skordas, E.S., 2002. Long-range correlations in the electric signals the precede rupture. Phys. Rev. E 66, 011902.

Varotsos, P.A., Sarlis, N.V., Skordas, E.S., 2003a. Attempt to distinguish electric signals of a dichotomous nature. Phys. Rev. E 68, 031106.

Varotsos, P.A., Sarlis, N.V., Skordas, E.S., 2003b. Long-range correlations in the electric signals the precede rupture: further investigations. Phys. Rev. E 67, 021109.

Varotsos, P.A., Sarlis, N.V., Tanaka, H.K., Skordas, E.S., 2005. Some properties of the entropy in the natural time. Phys. Rev. E 71, 032102.

Varotsos, P.A., Sarlis, N.V., Skordas, E.S., Tanaka, H.K., Lazaridou, M.S., 2006a. Attempt to distinguish long-range temporal correlations from the statistics of the increments by natural time analysis. Phys. Rev. E 74, 021123.

Varotsos, P.A., Sarlis, N.V., Skordas, E.S., Tanaka, H.K., Lazaridou, M.S., 2006b. Entropy of seismic electric signals: analysis in the natural time under time reversal. Phys. Rev. E 73, 031114.

Varotsos, P.A., Sarlis, N.V., Skordas, E.S., 2009. Detrended fluctuation analysis of the magnetic and electric field variations that precede rupture. Chaos 19, 023114.

Weinzierl, S., 2000. Introduction to Monte Carlo Methods. arXiv:hep-ph/0006269v1. 